\title{
Instantons and Algebraic Geometry
}

\author{
M. F. Atiyah and R. S. Ward \\ Mathematical Institute, University of Oxford, Oxford OX1 3LB, England
}

\begin{abstract}
Minimum action solutions for SU(2) Yang-Mills fields in Euclidean 4space correspond, via the Penrose twistor transform, to algebraic bundles on the complex projective 3-space. These bundles in turn correspond to algebraic curves. The implication of these results for the Yang-Mills fields is described. In particular all solutions are rational and can be constructed from a series of Ansätze $A_{l}$ for $l \geqq 1$.
\end{abstract}

\section{§ 1. Introduction}

The term instanton or pseudo-particle has been coined for the minimum action solutions of SU(2) Yang-Mills fields in Euclidean 4-space $R^{4}$. Conditions at infinity are imposed which are tantamount to working on the 4-sphere $S^{4}$ (which is the conformal compactification of $R^{4}$ ) and are classified by an integer $k$, which is interpreted as the "number of instantons". The most general solutions so far constructed explicitly are those of Jackiw et al. [4]. They depend on $5|k|+4$ real parameters if $|k| \geqq 3$, while for $|k|=1,2$ the number of parameters are 5,13 respectively. On the other hand infinitesimal deformation theory shows that the number of parameters for the complete family of solutions is $8|k|-3$ (see $[1,5,9]$ ). The purpose of this note is to describe how the full $(8|k|-3)$-parameter family can in principle be constructed by using algebraic geometry.

The basic idea is to use the Penrose Twistor approach to space-time [8] in which field equations in 4-space are converted into complex analytic geometry on complex projective 3-space $P_{3}$. This approach was applied in [11] to the self-dual (or antiself dual) Yang-Mills equations (which correspond to minimum action). The resulting geometrical objects on $P_{3}$ turn out to be complex analytic bundles. This transformation can be applied both locally and globally and for either Minkowski or Euclidean space. For the instanton problem we take the global Euclidean version (i.e. for $S^{4}$ ) and this leads to a complex analytic bundle defined over the whole of $P_{3}$. By Serre's basic theorems of analytic geometry [10] such bundles are necessarily algebraic. Moreover algebraic geometers have recently made significant progress on the study of precisely those algebraic bundles which correspond to the SU(2) Yang- 
Mills equations $[2,3,6]$. We shall apply these results and interpret them back in the Euclidean picture.

From a computational point of view the conclusion may be described briefly as follows. The instanton solutions so far known have been constructed by an Ansatz due to t'Hooft. This Ansatz which we shall label $A_{1}$ starts from a suitable solution of the (linear) Laplace equation in 4-space. From the algebraic geometry we find that there is a whole hierarchy of Ansätze $A_{l}(l=1,2, \ldots)$. The Ansatz $A_{2}$ starts from a solution of the (linear) anti-self dual Maxwell equations while $A_{l}$ for $l \geqq 3$ uses the corresponding first order equations for fields of spin $(l-1)$. The solutions obtained from $A_{l}$ are included in those coming from $A_{l+1}$ but not vice-versa in general. For $k=1,2$ the Ansatz $A_{1}$ suffices to give ( $\left.8 k-3\right)$-parameters but for $k=3,4$ we must use $A_{2}$. For any given $k$ there exists a large integer $l(k)$ so that all solutions come from $A_{l(k)}$. There are conjectural values of $l(k)$ but these are not yet established.

For solutions to be globally well-defined on $S^{4}$ the solutions of the linear equations have to be suitably chosen. For $A_{1}$ the appropriate solution $\phi$ of the Laplace equation $\Delta \phi=0$ has $\frac{1}{r^{2}}$ singularities at a given set of points. For $A_{2}$ the appropriate solution of the anti-self-dual Maxwell equations has a singularity along a 2-dimensional surface in $R^{4}$. However this surface is not arbitrary: it corresponds to an elliptic curve in $P_{3}$. Topologically it is a torus (with some self-crossing, i.e. double points) but analytically it is constrained to satisfy a certain differential equation which can be described in terms of its second fundamental form.

The algebraic character of our solutions is reflected in the fact that the field $F$ on 4-space is, in a suitable gauge, given by rational functions. In particular the action density is rational and its poles in the complex domain play an important role.

In this note detailed mathematical arguments will not be given. Instead we concentrate on describing the conclusions. A fuller treatment which necessarily involves extensive use of modern algebraic geometry will appear elsewhere.

Acknowledgment. We are much indebted to I.M.Singer for interesting us in Yang-Mills fields and to R.Penrose, N.J.Hitchin, and R.Hartshorne for many stimulating and useful discussions.

\section{§2. The Penrose Transform}

In this section we shall describe briefly how the Penrose theory leads to a reinterpretation of the self-dual Yang-Mills equations. Penrose's starting point is the observation that the complexification $Q_{4}$ of $S^{4}$ given by the homogeneous equation $\sum_{1}^{5} z_{i}^{2}=z_{6}^{2}$ in $P_{5}$ can be identified with the Grassmannian of lines in $P_{3}$. This goes back to Felix Klein and is based on the Plücker coordinates $\pi_{\alpha \beta}=x_{\alpha} y_{\beta}-x_{\beta} y_{\alpha}$ of the line $x, y$ in $P_{3}$. The $\pi_{\alpha \beta}$ are skew-symmetric, determined up to a scalar and satisfy the quadratic identity

$$
\pi_{01} \pi_{23}+\pi_{02} \pi_{31}+\pi_{03} \pi_{12}=0
$$

which by a suitable complex change of coordinates is identified with the equation $\sum_{1}^{5} z_{i}^{2}=z_{6}^{2}$ of $Q_{4}$. One now transforms problems on $S^{4}$ by first complexifying them to $Q_{4}$ and then using the Klein correspondence to pass to $P_{3}$. Applying this Penrose 
transform to a solution of the self-dual SU(2) Yang-Mills equations on $S^{4}$ one finds quite easily (see [11]) that the corresponding object is a 2-dimensional complex analytic (and therefore algebraic) vector bundle $E$ over $P_{3}$. Note that this bundle has only its complex structure it does not have a connection. The data of the Yang-Mills connection has become coded into pure complex structure. The fact that we work with the real group $\mathrm{SU}(2)$ and not just with its complexification $\operatorname{SL}(2, C)$ leads to some real algebraic constraints on $E$ which we shall now describe.

It is convenient to introduce the quaternions $H$, obtained by adjoining an element $j$ to the complex field $C$ : we take $j$ to satisfy $j^{2}=-1, j i=-i j$. Then $H=C+C j \cong C^{2}$ and so $H^{2} \cong C^{4}$. Left multiplication by $j$ on $H^{2}$ then induces an anti-linear map $\sigma: P_{3} \rightarrow P_{3}$ with $\sigma^{2}=1$. This defines a "real structure" on $P_{3}$ different from the usual real structure in which $\sigma$ would be the standard complex conjugation. In terms of Lie groups the standard real structure corresponds to the real form $\operatorname{SL}(4, R)$ of SL $(4, C)$ while the real structure given by $\sigma$ above corresponds to the real form $\mathrm{SO}(5,1)$ of $\mathrm{SO}(6, C)$ : recall that $\operatorname{Spin}(6, C) \cong \mathrm{SL}(4, C)$. In other words the subgroup of $\operatorname{SL}(4, C)$, acting on $P_{3}$, which commutes with $\sigma$ is $\operatorname{Spin}(5,1)$, the double cover of $\mathrm{SO}(5,1)$. Note that $\sigma$ has no fixed points i.e. there are no "real points" in $P_{3}$ but it has fixed lines and these are naturally parametrized by the 1-dimensional $H$-subspaces of $H^{2}$ which correspond to points of $H \cup \infty=R^{4} \cup \infty=S^{4}$. Spin $(5,1)$ acts on this $S^{4}$ as (the double cover of) the conformal group. Note that a line fixed by $\sigma$ (which we shall call a "real line") is a 2-sphere with $\sigma$ being the anti-podal map.

If $\sigma$ on $P_{3}$ can be lifted to an anti-linear involution (still called $\sigma$ ) on a vector bundle $E$ we say that $E$ has a real structure. Concretely this means that we have, for each $x \in P_{3}$, a linear isomorphism $\sigma_{x}: E_{x} \rightarrow \overline{E_{\sigma(x)}}$ varying algebraically (or holomorphically) with $x$ and satisfying $\sigma^{2}=1$. If $\sigma^{2}=-1$ we say $E$ has a symplectic structure. Note that the standard (Hopf) line-bundle $L$ over $P_{3}$ has a symplectic structure given by the quaternion $j$, but that $L^{2}=L \otimes L$ then has a real structure. More generally if $E$ has a real structure $E \otimes L$ is symplectic and vice-versa.

The constraints which we want to impose on a 2-dimensional algebraic vector bundle over $P_{3}$ are now:

1) $E$ has a symplectic structure.

2) The restriction of $E$ to every real line of $P_{3}$ is (algebraically) trivial.

To understand the significance of (2) we recall that algebraic 2-dimensional bundles over a projective line are isomorphic to a direct sum $L_{1} \oplus L_{2}$ of linebundles, the $L_{i}$ being essentially unique and each is determined by an integer $k_{i}$. The sum $k_{1}+k_{2}$ is a topological invariant called the first Chern class (hence will be constant for the restriction of $E$ to any line in $P_{3}$ ). However the difference $k_{1}-k_{2}$ can jump under small deformations. Under the assumption (2) $k_{1}=k_{2}=0$ for all real lines of $P_{3}$, hence (by semi-continuity) for the general line. However there will be an exceptional set of so-called jumping lines for which $k_{1} \neq k_{2}$.

The precise reformulation of SU(2) Yang-Mills is then given by:

Theorem. There is a natural one-one correspondence between

a) self-dual solutions $A$ of the $\mathrm{SU}(2)$ Yang-Mills equations on $S^{4}$ up to gauge equivalence and

b) isomorphism classes of 2-dimensional algebraic vector bundles $E$ over $P_{3}$ satisfying conditions (1) and (2) above. 
Notes. (i) The instanton number $k$ of $A$ corresponds to the only topological invariant of $E$ [called the second Chern class $\left.c_{2}(E)\right]$.

(ii) To apply the Penrose transform one needs to assume that $A$ is real-analytic. However one can give an alternative differential geometric approach which turns the self-dual Yang-Mills equations on $S^{4}$ into the integrability conditions for a complex analytic structure on $E$ and then apply the Newlander-Nirenberg theorem [7] which only requires mild differentiability. Thus analyticity is an automatic consequence.

(iii) If we replace condition (1) by the assumption that $E$ has a real structure, then we are led to self-dual $\operatorname{SL}(2, R)$ Yang-Mills fields. This is the other "real form".

The correspondence in the theorem is itself algebraic. Hence as a first Corollary we deduce that any self-dual Yang-Mills field is itself rational (in a suitable gauge) and that the action density is rational. Its poles in the complexification $Q_{4}$ lie on the hypersurface $f\left(z_{1}, \ldots, z_{6}\right)=0$ corresponding to the jumping lines of $E$. Moreover a result of Barth [2] shows that $f$ is a polynomial of degree exactly $k$ (the instanton number).

\section{§3. Algebraic Geometry of Bundles on $\boldsymbol{P}_{3}$}

We shall now review rapidly the main results concerning algebraic 2-dimensional vector bundles over $P_{3}$. There is a good theory only for those bundles known as stable bundles. These can be characterized by the property that any algebraic endomorphism of $E$ (i.e. a linear map $E_{x} \rightarrow E_{x}$ varying algebraically with $x$ ) is a constant scalar. Fortunately the bundles $E$ occurring in the theorem are easily seen to have this property, unless $k=0$ in which case $A$ is a pure gauge and $E$ is trivial.

A bundle $E$ on $P_{2}$ has 2 integer topological invariants, its Chern classes $c_{1}(E)$, $c_{2}(E)$. For our bundles $c_{1}(E)=0$ because of condition (2) [this corresponds to passing from $\operatorname{GL}(2, C)$ to $\operatorname{SL}(2, C)]$. This leaves $k=c_{2}(E) \geqq 0$ : we assume $k>0$. The first general result due to Maruyama (for preliminary results see [6]) is that the isomorphism classes of all stable 2-dimensional algebraic vector bundles over $P_{3}$ with $c_{1}=0, c_{2}=k$ are naturally parametrized by points of a (possibly reducible) algebraic variety $M(k)$. The real structure $\sigma$ of $P_{3}$ induces a real structure $\sigma$ on $M(k)$ [associate to $E$ the bundle $\sigma^{*}(\bar{E})$, whose fibre at $x \in P_{3}$ is $\bar{E}_{\sigma(x)}$ ]. The real points $M(k)_{R}$ of $M(k)$, namely those fixed by $\sigma$, represent either real or symplectic bundles and the action of $\sigma$ on $E$ is then unique (up to \pm 1 ). Moreover the condition $\sigma^{2}=+1$ or $\sigma^{2}=-1$ must be constant on connected components of $M(k)_{R}$. Thus we have a disjoint union

$$
M(k)_{R}=M(k)_{R}^{+} \cup M(k)_{R}^{-}
$$

where $M(k)_{R}^{-}$represents the symplectic bundles $\left(\sigma^{2}=-1\right)$ while $M(k)_{R}^{+}$represents the real bundles $\left(\sigma^{2}=1\right)$.

Finally the condition (2) of $\S 2$ defines an open set of $M(k)_{R}^{-}$. We know this set is non-empty because self-dual solutions have been constructed for every $k$. By the results of [1] we know that this open set of $M(k)_{R}^{-}$is a real manifold of dimension $(8 k-3)$. Because it is given by algebraic conditions it has a finite number of components. 
Complete information on the space $M(k)$ is not yet available but quite a lot is known. In describing this we will' for simplicity work only over the complex field, omitting any reference to the real (or unitary) restriction.

If $E$ is a stable bundle with $c_{1}(E)=0, c_{2}(E)=k$ then for some sufficiently large integer $l$ (depending on $k$ ) $E(l)=E \otimes L^{l}$ will have an algebraic section $s$ vanishing along a non-singular algebraic curve $\Gamma \subset P_{3}$. If $\Gamma$ is connected it uniquely determines $E$ (up to isomorphism) while if $\Gamma$ has (disjoint) components $\Gamma_{1}, \ldots, \Gamma_{r}$ we need to specify certain non-zero constants $c_{1}, \ldots, c_{r}$ (up to a common multiple) in order to determine $E$. Thus every $E$ arises from some $l$, some $\Gamma$ and some $\left(c_{1}, \ldots, c_{r}\right)$. However there is much redundancy here because $E(l)$ will in general have many sections (the number increases with $l$ ) and hence the same bundle $E$ arises from many different curves. In particular $E$ is trivial (and the associated Yang-Mills field is zero) if and only if $\Gamma$ is the complete intersection of two algebraic surfaces. If $E(l)$ has an unexpected section, i.e. when $l$ is small compared with $k$, then this section is likely to be unique and is an invariant of the bundle. This happens for $l=1$ and $k \geqq 3$ with the solutions of [4].

The component curves $\Gamma_{i}$ are not arbitrary. First of all we have the following numerical formulae for the degree $d_{i}$ and the genus $g_{i}$ :

$$
\begin{gathered}
\sum_{1}^{r} d_{i}=k+l^{2} \\
g_{i}-1=(l-2) d_{i} .
\end{gathered}
$$

Moreover the second of these, when multiplied by 2 , underlies an equality between divisor classes on $\Gamma_{i}$, namely $K=2(l-2) D_{i}$ where $K$ is the canonical class of $\Gamma_{i}$ and $D_{i}$ is the class of a plane section. If $g_{i}=0$ or 1 this gives no additional restriction but for $g_{i}>1$ it is a severe restraint on the kind of curve which can occur.

The simplest solutions of the above equations are given by $l=1, d_{i}=1, g_{i}=0$. This means that each $\Gamma_{i}$ is a projective line. These correspond precisely to the known solutions of [4], provided we take the lines to be "real" i.e. corresponding to points of $S^{4}$. Note that the number of points is $r=k+1$.

The next simplest solutions are for $l=2, g_{i}=1$. The $\Gamma_{i}$ are now elliptic curves of degree $d_{i}$ where $\Sigma d_{i}=k+4$. For example, taking $r=1$, we can take a single elliptic curve of degree $k+4$. The bundle $E$ defined by such an elliptic curve $\Gamma$ coincides with the bundle defined by $k+1$ disjoint lines if and only if $\Gamma$ lies on a cubic surface. For $k=1,2$ every such $\Gamma$ can be shown to have this property but for $k \geqq 3$ only a subset of $\Gamma$ lie on cubic surfaces. For $k=3$ one finds that elliptic curves of degree 7 give a 21-parameter family of bundles whereas the elliptic curves lying on cubic surfaces depend on two parameters less and give only a 19-parameter family of bundles.

The disjoint addition of curves $\Gamma_{i}$, generalizing the case of lines, can be viewed as some form of superposition principle for the nonlinear differential equations of the Yang-Mills theory. This geometrical viewpoint may help to cast light on the whole "soliton" phenomenon.

For $k=1$ results of Barth [2] imply that the only bundles on $P_{3}$ are the nullcorrelation bundles. These correspond to the SU(2)-bundles on $S^{4}$ which are homogeneous under $\mathrm{SO}(5)$ and give rise to the standard instanton. Thus the 
parameter space for self-dual solutions in this case is just the hyperbolic 5-space (interior of $S^{4}$ ). For $k>1$ it is not yet known whether the parameter space is connected.

\section{§4. The Ansatz $A_{l}$}

If $s$ is a section of $E(l)$ vanishing along $\Gamma$ as above then on $P_{3}-\Gamma$ the bundle $E(l)$ has a non-vanishing section which therefore generates a trivial line-bundle, and $E(l)$ is an extension of this by the line-bundle $L^{2 l}$. Extensions of this type are classified by elements of the first sheaf cohomology group $H^{1}\left(P_{3}-\Gamma, \mathcal{O}(-2 l)\right)$ where $\mathcal{O}(-2 l)$ denotes the sheaf of algebraic sections of $L^{-2 l}$ (the dual of $L^{2 l}$ ). In more computational terms the existence of a non-vanishing section $s$ of $E$ means that the transition matrices of $E$, which normally lie in $\operatorname{SL}(2, C)$, can be put in triangular form

$$
\left(\begin{array}{ll}
1 & a \\
0 & b
\end{array}\right)
$$

in a suitable gauge (on $P_{3}-\Gamma$ ). The elements $b$ can be standardized and correspond to the line-bundle $L^{2 l}$ while the elements $a$ correspond to the element of $H^{1}\left(P_{3}-\Gamma, \mathcal{O}(-2 l)\right)$. In the Penrose transform one knows [8] that elements of $H^{1}\left(P_{3}-\Gamma, \mathcal{O}(-2 l)\right)$ correspond naturally to anti-self-dual solutions $\phi$ of the Maxwell equations for $\operatorname{Spin}(l-1)$ fields (if $l>1)$ and to scalar densities satisfying the Laplace equation if $l=1$. These solutions $\phi$ have singularities on the surface $\tilde{\Gamma}$ in $S^{4}$ which corresponds to $\Gamma$. If one examines the details of the Penrose transform one can in principle describe explicitly an Ansatz $A_{l}$ that associates to each $\phi$ a solution of the self-dual $(\operatorname{SL}(2, C))$ Yang-Mills equations. We shall do this for the cases $l=1$ and $l=2$.

It is convenient to use an index notation ${ }^{1}$. Let $\left\{P_{1 \mu \nu}, P_{2 \mu \nu}, P_{3 \mu \nu}\right\}$ be an orthonormal basis for the space of anti-self-dual 2-forms on $R^{4}$ (thus $* P_{j \mu \nu}=-P_{j \mu v}$, $\left.P_{j}^{\mu v} P_{k \mu \nu}=\delta_{j k}\right)$. The $l=1$ Ansatz is the known one of [4]; it can be written

$$
A^{\mu}=\frac{1}{2 i} A_{j}^{\mu} \sigma^{j}
$$

where $A_{j}^{\mu}=2 P_{j}^{\mu \nu} \nabla_{v} \log \phi$, with $\Delta \phi=0$. On $S^{4}, \phi$ has to be interpreted as a scalar density and the Laplace operator $\Delta$ has to be replaced by its conformally invariant analogue.

The $l=2$ Ansatz may be described as follows.

Define

$$
\begin{aligned}
& R_{1}^{\mu \nu \alpha \beta}=i\left(P_{1}^{\mu \nu} P_{2}^{\alpha \beta}+P_{2}^{\mu \nu} P_{1}^{\alpha \beta}\right), \\
& R_{2}^{\mu \nu \alpha \beta}=i\left(P_{2}^{\mu \nu} P_{2}^{\alpha \beta}-P_{1}^{\mu \nu} P_{1}^{\alpha \beta}\right), \\
& R_{3}^{\mu \nu \alpha \beta}=-P_{3}^{\mu \nu} P_{3}^{\alpha \beta} .
\end{aligned}
$$

Let $\phi_{\mu \nu}$ be an anti-self-dual solution of Maxwell's equations, and write $\phi^{2}=\phi_{\mu \nu} \phi^{\mu \nu}$.

1 The Greek indices $\mu, v, \alpha, \beta, \ldots$ are 4-space indices, whereas $j, k$ range over $1,2,3$. The three Pauli matrices are denoted $\sigma^{j}$. The Einstein summation convention is employed, and square brackets enclosing indices denote skew-symmetrization 
Then

$$
A_{j}^{\mu}=2 R_{j}^{\mu \nu \alpha \beta} \phi^{2}\left(\phi_{\varrho \sigma} P_{3}^{\rho \sigma}\right)^{-1} \nabla_{v}\left(\phi^{-2} \phi_{\alpha \beta}\right)
$$

is a self-dual solution of the Yang-Mills equations.

Note that the $R_{j}^{\mu \nu \alpha \beta}$ are complex : so the Maxwell field has to be complex if the $A_{j}^{\mu}$ are to have a chance of being real. This is a feature of the Ansätze $A_{l}$ for even values of $l$ : the reality conditions are trickier to describe and are not built into the Ansatz in a manifest way.

Both for $l=1$ and $l=2$ the Ansatz leads to a potential $A_{j}^{\mu}$ which has apparent singularities. These arise from singularities of $\phi$ and from zeros of $\phi$ (in case $l=1$ ) and of $\phi^{2}$ (in case $l=2$ ). The singularity (for $l=2$ ) arising from real zeros of $\phi_{\rho \sigma} P_{3}^{\rho \sigma}$ appears to be a gauge artifact. For $l=1$ poles of $\phi$ of the form $\frac{1}{r^{2}}$ give a potential whose corresponding singularities are removable by a gauge transformation: the zeros are then kept off the real domain by taking $\phi$ positive as in [4]. For $l=2$ nonsingular self-dual solutions of the Yang-Mills equations arise, as explained earlier, from elliptic curves $\Gamma$ in $P_{3}$. This means that $\phi$ must be rational and its real poles must be located on the corresponding surface $\tilde{\Gamma}$ in $S^{4}$ and be of a suitable type. Moreover $\phi^{2}$ must have no real zeros.

The exact "type of singularity" of $\phi$ along $\tilde{\Gamma}$ has yet to be made explicit. However the case of $l=1$ and algebraic geometric considerations strongly suggest that $\phi$ should be defined as a distributional solution of the inhomogeneous anti-self-dual Maxwell equations with an appropriate Dirac delta function along $\tilde{\Gamma}$ as right-hand side. The detailed working out of this idea will require extending the theory of the Penrose transform so as to provide a translation of language between homological algebra in $P_{3}$ and distribution theory in $S^{4}$.

As an illustration of the formulae we consider the simple case when $\Gamma$ is a disjoint union of elliptic quartic curves $\Gamma_{i}, i=1,2, \ldots, r$. Each such quartic curve is the intersection of two quadric surfaces in $P_{3}$. A quadric in $P_{3}$, together with a scale factor, corresponds to an anti-self-dual 2-form $q_{\mu \nu}$ in $R^{4}$ of the form

$$
\begin{aligned}
q_{\mu \nu}= & 4 B_{\alpha[\mu} x^{\alpha} x_{v]}+x^{2} B_{\mu \nu}+2 C_{[\mu} x_{v]} \\
& -\varepsilon_{\mu \nu \alpha \beta} C^{\alpha} x^{\beta}+D_{\mu \nu},
\end{aligned}
$$

where $B_{\mu v}\left(D_{\mu v}\right)$ are constant self-dual (anti-self-dual) 2-forms and $x^{2}=x_{\mu} x^{\mu}$ (cf. Equation (4.12) of [4]). So $q_{\mu v}$ is determined by 10 complex parameters.

If $Q$ is another quadric in $P_{3}$ and $Q_{\mu v}$ is the corresponding 2-form in $R^{4}$, then the Maxwell field corresponding to the elliptic quartic curve $q \cap Q$ is

$$
\phi_{\mu \nu}=\left(Q_{\beta[\gamma} q_{\delta]}^{\beta} Q_{\varrho}^{\gamma} q^{\rho \delta}\right)^{-1} Q_{\alpha[\mu} q_{v]}^{\alpha} .
$$

The Maxwell fields corresponding to $r$ disjoint elliptic quartics are obtained by simply taking linear combinations of the Maxwell fields obtained from each one. Note that, for $r=1, \Gamma$ is a complete intersection so that the potential $A_{\mu}^{j}$ will be a pure gauge, but this is no longer the case for $r \geqq 2$.

Remark. The difference between self-dual and anti-self-dual is a matter of orientation of 4-space. There is thus no essential difference between the two cases but once an orientation has been chosen we must stick with it. The important thing about our Ansatz $A_{l}$ is that it associates, to a solution of the linear Maxwell equations of one type of duality, a solution of the non-linear Yang-Mills equations 
of the opposite type of duality. This switch is of course not apparent for the case $l=1$.

The surface $\tilde{\Gamma}$ which carries the singularities of $\phi$ is the image of $\Gamma$ under a continuous map. This map $\tilde{\Gamma}$ may either be $1-1$ in general, in which case $\tilde{\Gamma}$ is orientable, or $2-1$ in general in which case $\tilde{\Gamma}$ is non-orientable. In the orientable case one can also show that $\tilde{\Gamma}$ has precisely $k$ double points or self-crossings, where $k$ is the instanton number of the Yang-Mills field.

\section{§5. Further Comments}

Although the algebraic geometry provides in theory a complete solution for the selfdual Yang-Mills fields, there are a number of serious difficulties involved. First of all the curves which correspond to $l \geqq 3$ are of high genus and very special. Not much is known about how to construct them, whereas for $l=2$ the elliptic curves can be quite explicitly described.

Secondly the reality conditions which require the complex singularities to avoid $S^{4}$ are not easy to work with. In geometric terms the jumping lines of a bundle on $P_{3}$ are hard to identify.

It may turn out that the reality constraints just mentioned can be put to constructive use and may help in the classification problem, but this is speculative.

If we move from SU(2) to $\mathrm{SU}(3)$ then the Penrose transform still converts the problem into one on $P_{3}$ but as yet the algebraic-geometric results here are rudimentary. It is perhaps interesting to observe that $\mathrm{SU}(n)$ for $n \geqq 4$ would lead to $n$-dimensional bundles on $P_{3}$ and that these can in a sense be reduced down to $n=3$, i.e. every $n$-dimensional bundle for $n \geqq 4$ is an extension of a 3-dimensional bundle by a sum of line-bundles.

Finally we should point out that by working on $S^{4}$ instead of $R^{4}$ we are making an assumption about the asymptotic behaviour of the Yang-Mills field which may be technically stronger than convergence of the action integral. This point deserves further investigation and it can be studied via the Penrose transform where it concerns the behaviour of a complex analytic bundle on $P_{3}-P_{1}$, the line $P_{1}$ corresponding to the point at infinity of $R^{4}$.

\section{References}

1. Atiyah,M.F., Hitchin, N.J., Singer,I.M. : Proc. Nat. Acad. Sci. U.S. 74 (1977)

2. Barth,W.: Math. Ann. 226, 125-150 (1977)

3. Grauert,H., Mülich,G. : manuscripta math. 16, 75-100 (1975)

4. Jackiw, R., Nohl,C., Rebbi,C.: Phys. Rev. D 15, 1642-1646 (1977)

5. Jackiw, R., Rebbi,C.: Phys. Letters 67 B, 189-192 (1977)

6. Maruyama,M. : Nagoya Math. J. 58, 25-68 (1975)

7. Newlander, A., Nirenberg,L.: Ann. Math. 65, 391-404 (1957)

8. Penrose, R.: The twistor programme. Rept. Math., Phys., to appear

9. Schwarz, A. S. : Phys. Letters 67B, 172-174 (1977)

10. Serre,J.P.: Ann. Inst. Fourier VI, $1-42$ (1956)

11. Ward, R.S. : Phys. Letters 61 A, 81-82 (1977)

12. Witten,E.: Some exact multi-instanton solutions of classical Yang-Mills theory (Preprint, 1976)

Communicated by R. Stora

Received May 31, 1977 